\title{
Electron induced light emission in photonic crystals
}

\author{
L. A. Blanco ${ }^{1, *}$ and F. J. García de Abajo ${ }^{1,2}$ \\ ${ }^{1}$ Donostia International Physics Center (DIPC), \\ Aptdo. 1072, 20080 San Sebastián, Spain \\ ${ }^{2}$ Centro Mixto CSIC-UPV/EHU, \\ Aptdo. 1072, 20080 San Sebastián, Spain
}

(Dated: October 30, 2018)

\begin{abstract}
ID:A1857

The interaction of a fast electron with a photonic crystal is studied by solving the Maxwell equations exactly for the external field provided by the electron in the presence of the crystal. The polarization currents and charges produced by the passage of the electron give rise to the emission of the so-called Smith-Purcell radiation. The emitted light probability is obtained by integrating the Poynting vector over planes parallel to the crystal at a large distance from the latter. Both reflected and transmitted light components are analyzed and related to the photonic band structure of the crystal. Emission spectra are compared with the energy loss probability and also with the reflectance and transmittance of the crystal.
\end{abstract}

Keywords: Electron solid interactions, photonic crystals, electroluminiscence.

\footnotetext{
* phone number: ++34943015421

fax number: ++34943015600

e-mail address: lablancoj@sc.ehu.es
} 


\section{INTRODUCTION}

Photonic crystals have received considerable attention as many technological applications are being proposed including control of atomic and molecular spontaneous emission [1] or

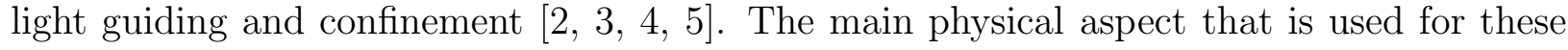
purposes is the existence of photonic bandgaps in such systems, that can lie in the near infrared [6, 7, 8] or in the visible [9], which allow light to follow a determined path depending on the structure of the crystal. Despite recent advances in the production of photonic crystals that operate in both regions of the light spectrum [10], fabrication defects can still limit their applicability. However, a quantitative determination of the number and type of the defects in a crystal is difficult to perform. Electrons seem to be a valid probe to that end. Here, we study the interaction of a fast electron with a crystal as a first approach to this problem.

In particular, a fast electron moving near a photonic crystal can polarize its constituents inducing charges and currents that cannot follow the electron motion and suffer acceleration as they evolve in the inhomogeneous crystal, giving rise to light emission. This is similar to the so-called Smith-Purcell effect [11, 12, 13, 14, 15, 16, 17].

The present work is intended to provide a new way to characterize photonic crystals by observing the spectrum of the light emitted when electrons are travelling parallel to the surface of the crystal. The electric field of the moving electron is decomposed into evanescent plane waves [18], and the outgoing light is produced by the diffraction of these waves in the crystal. This procedure is sketched in Section II. Numerical results for a complete-band-gap photonic crystal formed by air spheres in silicon (inverted opal) are shown in Section III. We use electrons travelling within air and silicon, in order to reflect different electrodynamic effects, although a possible experimental observation could be more difficult in the latter case. Gaussian atomic units will be used from now on, unless otherwise specified.

\section{THEORETICAL FRAMEWORK}

A theoretical description of the interaction between an electron and a crystal is given next. The crystal will be composed of several layers perpendicular to the $z$ direction, beginning in $z=0$ and extending towards the negative- $z$ region. Outside the crystal a medium with a dielectric function $\epsilon$ will also be considered. All media will be assumed to be non-magnetic 
$(\mu=1)$.

The electron will be considered to follow a trajectory described by $\mathbf{r}_{t}=\left(v t, y_{0}, z_{0}\right)$, with $z_{0}>0$, and its electric field is found to be, in frequency space $\omega$ and in the absence of the crystal,

$$
\mathbf{E}_{0}(\mathbf{r}, \omega)=\int d Q_{y} \mathrm{e}^{\left.\mathrm{i} \mathbf{K}_{\mathbf{Q}}^{ \pm} \cdot\left[\mathbf{r}-\left(0, y_{0}, z_{0}\right)\right]\right)} \mathbf{E}_{\mathbf{Q}}^{ \pm},
$$

where $\mathbf{K}_{\mathbf{Q}}^{ \pm}=\left(\mathbf{Q}, \pm \mathrm{i} \Gamma_{\mathbf{Q}}\right), \mathbf{Q}=\left(\omega / v, Q_{y}\right)$ is the two-dimensional momentum parallel to the surface, and $\Gamma_{\mathbf{Q}}^{2}=Q^{2}-\omega^{2} \epsilon / c^{2}$, with $\operatorname{Re}\left\{\Gamma_{\mathbf{Q}}\right\}>0$. In this expression, $\mathbf{E}_{\mathbf{Q}}^{ \pm} \exp \left(\mathrm{i} \mathbf{K}_{\mathbf{Q}}^{ \pm} \cdot \mathbf{r}\right)$ is a plane wave, which can be expressed as a sum of $s$ and $p$ components, $\mathbf{E}_{\mathbf{Q}}^{ \pm}=E_{\mathbf{Q}, s}^{ \pm} \hat{\mathbf{e}}_{\mathbf{Q}, s}^{ \pm}+$ $E_{\mathbf{Q}, p}^{ \pm} \hat{\mathbf{e}}_{\mathbf{Q}, p}^{ \pm}$. The $+(-)$sign in these expressions stands for a wave moving towards the positive(negative-) $z$ region. When the electron moves in vacuum, all of these waves are evanescent ( $\Gamma_{\mathbf{Q}}$ is real and positive). However, when it moves in a medium described by $\epsilon>0$ and $v \sqrt{\epsilon}>c$, some of those waves represent propagating Cherenkov radiation.

Due to the crystal symmetry, an incident wave with momentum $\mathbf{K}_{\mathbf{Q}}^{-}$will only produce a discrete set of reflected (transmitted) waves of momentum $\mathbf{K}_{\mathbf{Q}+\mathbf{G}}^{+}\left(\mathbf{K}_{\mathbf{Q}+\mathbf{G}}^{-}\right), \mathbf{G}$ being a reciprocal surface lattice vector. Therefore, the reflected and transmitted wave amplitudes can be expressed as

$$
\begin{aligned}
& {\left[E_{\mathbf{Q}+\mathbf{G}, \sigma}^{+}\right]^{r}=\sum_{\sigma^{\prime}} R_{\mathbf{Q G}}^{\sigma \sigma^{\prime}}\left[E_{\mathbf{Q}, \sigma^{\prime}}^{-}\right]^{i},} \\
& {\left[E_{\mathbf{Q}+\mathbf{G}, \sigma}^{-}\right]^{t}=\sum_{\sigma^{\prime}} T_{\mathbf{Q G}}^{\sigma \sigma^{\prime}}\left[E_{\mathbf{Q}, \sigma^{\prime}}^{-}\right]^{i},}
\end{aligned}
$$

where $\sigma$ and $\sigma^{\prime}$ run over polarizations $s$ and $p$, the super-indices $r, t$ and $i$ stand for reflected, transmitted and incident components, respectively, and $R_{\mathbf{Q G}}^{\sigma \sigma^{\prime}}$ and $T_{\mathbf{Q G}}^{\sigma \sigma^{\prime}}$ are the reflection and transmission coefficients of the crystal. These coefficients are calculated using the layer KKR method [19], in which the self-consistent electric field is constructed in terms of multipole expansions around the crystal objects by multiple scattering within each layer and later among layers.

Each of the incident plane waves in Eq. (1) gives rise to a set of reflected and transmitted waves whose amplitudes are obtained according to Eq. (2). The electric field in the positive- $z$ region can be constructed as the sum of $\mathbf{E}_{0}$ and the reflected field,

$$
\mathbf{E}(\mathbf{r}, \omega)=\mathbf{E}_{0}(\mathbf{r}, \omega)+\mathbf{E}_{r}(\mathbf{r}, \omega),
$$


where

$$
\mathbf{E}_{r}(\mathbf{r}, \omega)=\sum_{\mathbf{G}, \sigma \sigma^{\prime}} \int d Q_{y} \mathrm{e}^{\mathrm{i} \mathbf{K}_{\mathbf{Q}+\mathbf{G}}^{+} \cdot \mathbf{r}} R_{\mathbf{Q G}}^{\sigma \sigma^{\prime}}\left[E_{\mathbf{Q}, \sigma^{\prime}}^{-}\right]^{i} \hat{\mathbf{e}}_{\mathbf{Q}+\mathbf{G}, \sigma}^{+} .
$$

A similar expression is found for the transmitted electric field at the other side of the crystal (negative $z ' s$ ):

$$
\mathbf{E}_{t}(\mathbf{r}, \omega)=\sum_{\mathbf{G}, \sigma \sigma^{\prime}} \int d Q_{y} \mathrm{e}^{\mathrm{i} \mathbf{K}_{\mathbf{Q}+\mathbf{G}}^{-} \cdot \mathbf{r}} T_{\mathbf{Q G}}^{\sigma \sigma^{\prime}}\left[E_{\mathbf{Q}, \sigma^{\prime}}^{-}\right]^{i} \hat{\mathbf{e}}_{\mathbf{Q}+\mathbf{G}, \sigma}^{-} .
$$

The light emission probability per unit length is calculated by means of the projection of the Poynting vector $\mathcal{P}$ over the $z$ and $-z$ directions (for reflected and transmitted components, respectively), which is integrated over the time and over a plane parallel to the crystal surface. The resulting expression can be written

$$
\int d t \int d x \int d y \mathcal{P} \cdot( \pm \hat{z})=\int_{0}^{\infty} \omega d \omega P(\omega)
$$

where $P(\omega)$ can be interpreted as the emission probability per unit length and per energy range $\omega$. The average over parallel impact parameters $y_{0}$ has also been performed. When the Cherenkov condition $v^{2} \epsilon>c^{2}$ is not fulfilled, the direct waves of Eq. (1) are all evanescent, and the light emission by reflection and transmission at the crystal (i.e. emission towards the $z>0$ and $z<0$ regions, respectively denoted reflected emission and transmitted emission hereafter) reduces to

$$
\begin{aligned}
& P_{r}(\omega)=\frac{1}{2 \pi k^{2}} \sum_{\mathbf{G} \sigma}{ }^{\prime} \int d Q_{y}\left|r_{\mathbf{Q G} \sigma}\right|^{2} \Delta_{\mathbf{Q}+\mathbf{G}} e^{-2 \Delta_{\mathbf{Q}} z_{0}}, \\
& P_{t}(\omega)=\frac{1}{2 \pi k^{2}} \sum_{\mathbf{G} \sigma}^{\prime} \int d Q_{y}\left|t_{\mathbf{Q G} \sigma}\right|^{2} \Delta_{\mathbf{Q}+\mathbf{G}} e^{-2 \Delta_{\mathbf{Q}} z_{0}}
\end{aligned}
$$

where

$$
\begin{aligned}
r_{\mathbf{Q G} \sigma} & =\frac{\mathrm{i} Q_{y} \omega}{Q \Gamma_{\mathbf{Q}} c^{2}} R_{\mathbf{Q G}}^{\sigma s}-\frac{k}{v Q \sqrt{\epsilon}} R_{\mathbf{Q G}}^{\sigma p}, \\
t_{\mathbf{Q G} \sigma} & =\frac{\mathrm{i} Q_{y} \omega}{Q \Gamma_{\mathbf{Q}} c^{2}} T_{\mathbf{Q G}}^{\sigma s}-\frac{k}{v Q \sqrt{\epsilon}} T_{\mathbf{Q G}}^{\sigma p}, \\
\Delta_{\mathbf{Q}} & =\left|\Gamma_{\mathbf{Q}}\right|
\end{aligned}
$$

and the prime in the summation over $\mathbf{G}$ indicates that only non-evanescent outgoing waves $\left(\Gamma_{\mathbf{Q}+\mathbf{G}}^{2}<0\right)$ must be included. 
On the other hand, if $v^{2} \epsilon>c^{2}$, there exists an interference between the direct Cherenkov radiation and the reflected field. The emission probability expressions take a more involved form in this case:

$$
\begin{aligned}
P_{r}(\omega) & =\frac{1}{2 \pi k^{2}} \sum_{\sigma}\left\{\int_{-\infty}^{-Q_{0}} d Q_{y} \sum_{\mathbf{G}}{ }^{\prime}\left|r_{\mathbf{Q G} \sigma}\right|^{2} \Delta_{\mathbf{Q}+\mathbf{G}} e^{-2 \Delta_{\mathbf{Q}} z_{0}}+\right. \\
& +\int_{-Q_{0}}^{Q_{0}} d Q_{y}\left[\Delta_{\mathbf{Q}}\left|f_{\sigma}\right|^{2}+2 \operatorname{Re}\left(\Delta_{\mathbf{Q}} f_{\sigma} r_{\mathbf{Q} 0 \sigma} e^{-2 i \Gamma_{\mathbf{Q}} z_{0}}\right)+\sum_{\mathbf{G}}{ }^{\prime}\left|r_{\mathbf{Q G} \sigma}\right|^{2} \Delta_{\mathbf{Q}+\mathbf{G}}\right]+ \\
& \left.+\int_{Q_{0}}^{\infty} d Q_{y} \sum_{\mathbf{G}}{ }^{\prime}\left|r_{\mathbf{Q G} \sigma}\right|^{2} \Delta_{\mathbf{Q}+\mathbf{G}} e^{-2 \Delta_{\mathbf{Q}} z_{0}}\right\}, \\
P_{t}(\omega) & =\frac{1}{2 \pi k^{2}} \sum_{\sigma \mathbf{G}}{ }^{\prime}\left\{\int_{-\infty}^{-Q_{0}} d Q_{y}\left|t_{\mathbf{Q G} \sigma}\right|^{2} \Delta_{\mathbf{Q}+\mathbf{G}} e^{-2 \Delta_{\mathbf{Q}} z_{0}}+\right. \\
& +\int_{-Q_{0}}^{Q_{0}} d Q_{y}\left|t_{\mathbf{Q G} \sigma}\right|^{2} \Delta_{\mathbf{Q}+\mathbf{G}}+ \\
& \left.+\int_{Q_{0}}^{\infty} d Q_{y}\left|t_{\mathbf{Q G} \sigma}\right|^{2} \Delta_{\mathbf{Q}+\mathbf{G}} e^{-2 \Delta_{\mathbf{Q}} z_{0}}\right\},
\end{aligned}
$$

where $Q_{0}^{2}=k^{2} \epsilon-\omega^{2} / v^{2}, f_{p}=-k /(v Q \sqrt{\epsilon})$ and $f_{s}=\mathrm{i} Q_{y} \omega /\left(Q \Gamma_{\mathbf{Q}} c^{2}\right)$. The integrands in these expressions describe the reflected and transmitted wave components for external evanescent $\left(\left|Q_{y}\right|>Q_{0}\right)$ and propagating $\left(\left|Q_{y}\right|<Q_{0}\right)$ waves. In particular, the first term inside the square bracket of $P_{r}$ reduces to the Cherenkov emission probability in a bulk homogeneous medium, while the remaining terms describe reflected and interference components.

\section{RESULTS AND DISCUSSION}

We have applied the previous formalism to the case of a 100-keV electron moving parallel to a slab consisting on several fcc(100) and fcc(111) planes of air spheres in silicon. The gap characteristics of this system have been theoretically described in [23]: a complete photonic bandgap is opened when the ratio between the sphere radius $r$ and the cubic lattice constant $a$ lies in between 0.335 and 0.374 . We have chosen an intermediate value of $r / a=0.342$, and the frequency range is taken close to the communications wavelength, $\lambda=1.55 \mu \mathrm{m}$, in which case $\epsilon_{\mathrm{Si}}=11.9$.

Results for crystals composed of $8 \mathrm{fcc}(111)$ planes are shown in what follows, although we have also carried out calculations for $8 \mathrm{fcc}(100)$ planes and the results are qualitatively similar. Two different types of calculations are presented. a) In Fig. 1, the crystal is 
embedded in $\mathrm{Si}$, so that the electron moves within silicon outside the crystal. In this case, the electron produces Cherenkov radiation even without the presence of the crystal whenever the electron velocity is larger than the speed of light in the medium, which is the case at $100 \mathrm{keV}$. b) In Fig. 2, the crystal is embedded in air and the electron moves also outside the crystal, that is in air. Therefore, no direct Cherenkov radiation is produced.

As the total energy must be conserved in the present case in which the material is transparent (that is, $\epsilon$ is real) the sum of the reflected and transmitted probabilities must be the same as the electron energy loss probability, which has been calculated from the retarding force exerted on the electron by the induced electric field, as discussed elsewhere [20, 21].

The main difference between the two cases considered in Figs. 1 (a) and 2 is that when Cherenkov radiation is emitted the energy loss and the reflected emission are very much enhanced [Fig. 1 (a)]. This is due to the fact that the Cherenkov emission is dominant as compared to the diffraction of evanescent waves. For the transmitted light, as expected, the emission probability within the gap frequencies is strongly suppressed in both cases.

When the electron travels in air (Fig. 2), the energy loss and the reflected light probabilities are much smaller than in the previous case. However, the order of magnitude of the transmitted field is the same as in the Cherenkov case. Notice that huge peaks are produced in the reflection curve near the bandgap region, probably connected with band edge effects. This type of energy losses are well below the current energy resolution of STEM machines (around $0.5 \mathrm{eV}$ ), but since the sample is transparent, any energy loss must be converted into photon emission, for which the resolution is much better, as shown in [22], and sufficient to resolve the features discussed in this work. The integrated area of the sharp peaks in Fig. 2 amounts to approximately $5 \times 10^{-6}$ photons per electron per nm, which for path lengths of the order of a several microns, would result in a measurable photon intensity of a percentage of the number of electrons.

In both cases the gap regions agree reasonably well with those found for the transmission of external electromagnetic waves under normal and Cherenkov-angle incidence conditions as well as with the band structure of the system, which are shown for the first case (electron moving in Si) in Figs. 1 (b)-(c). Note that the transmitted emission gap [Fig. 1 (a)] is wider than the complete gap (vertical dashed lines throughout all the Fig. 1), since the latter corresponds to a particular selection of incident directions (the Cherenkov cone). The same is true for the transmittance [Fig. 1(b)]; actually, the case of normal incidence corresponds 
to the $\Gamma$ point. The depletion of light transmission within the gap could be used to detect defaults (stacking faults, missing atoms, etc.), whose relatively small contribution to the emission would be amplified because that would be the primary origin of light transmitted within the gap.

Finally, in Fig. 3 we show the dependence of the light emission probability on the distance between the electron and the crystal, for a given frequency within the bandgap. As expected, both the transmitted and reflected probabilities decrease when increasing the electron-crystal separation, but it is interesting how the magnitude of the interference between the direct (Cherenkov) and the reflected fields produces an oscillatory pattern at the front side of the crystal. For the transmitted field, the probability reaches an asymptotic value which comes from the transmission of only Cherenkov incident waves.

\section{Acknowledgments}

The authors gratefully acknowledge help and support from the Spanish Ministerio de Ciencia y Tecnología and the Basque Government. 
[1] V.P. Bykov, Sov. Phys. JETP 35, 269 (1972)

[2] E. Yablonovitch, Phys. Rev. Lett. 58, 2059 (1987).

[3] S. John, Phys. Rev. Lett. 58, 2486 (1987).

[4] J. Joannopoulos, R. Meade and J. Winn, Photonic Crystals, Princeton Press, Princeton, 1995.

[5] J.D. Joannopoulos, P.R. Villeneuve and S. Fan, Nature 386, 143 (1997).

[6] K.M. Ho, C.T. Chan and C.M. Soukoulis, Phys. Rev. Lett. 65, 3152 (1990).

[7] T.W. Ebbesen, H.J. Lezec, H.F. Ghaemi, T. Thio and P.A. Wolff, Nature 391, 667 (1998).

[8] M.S. Thijssen, R. Sprik, J.E.G.J. Wijnhoven, M. Megens, T. Narayanan, A. Lagendijk and W.L. Wos, Phys. Rev. Lett. 83, 2730 (1999).

[9] A. Moroz, Phys. Rev. Lett. 83, 5274 (1999).

[10] A. Blanco et al., Nature 405 (2000), 437.

[11] I.M. Frank, Izv. Akad. Nauk SSSR Ser. Fiz. 6, 3 (1942).

[12] S.J. Smith and E.M. Purcell, Phys. Rev. 92, 1069 (1953).

[13] J.P. Bachheimer, Phys. Rev. B 6, 2985 (1972).

[14] O. Haeberle, P. Rullhusen, J.-M. Salomé and N. Maene, Phys. Rev. E 49, 3340 (1994).

[15] J.B. Pendry and L. Martín-Moreno, Phys. Rev. B 50, 5062 (1994).

[16] F.J. García de Abajo, Phys. Rev. Lett. 82, 2776 (1999); Phys. Rev. E 61, 5743 (2000).

[17] K. Ohtaka and S. Yamaguti, Optics and Spectroscopy 91 (2001), 477.

[18] G. Toraldo di Franzia, Nuovo Cimento 16, 61 (1960).

[19] N. Stefanou, V. Yanopapas, and A. Modinos, Comput. Phys. Commun. 113 (1998) 49; 132 (2000) 189.

[20] F.J. García de Abajo and A. Howie, Phys. Rev. Lett. 80, 5180 (1998).

[21] F.J. García de Abajo and L.A. Blanco, in preparation.

[22] N. Yamamoto, K. Araya and F.J. García de Abajo, Phys. Rev. B 64, 205419 (2001).

[23] K. Busch and S. John, Phys. Rev. E 58, 3896 (1998). 
FIG. 1: (a) Frequency variation of the energy loss probability (thick solid curve), the reflected plus direct light emission probability (dashed curve) and the transmitted light emission probability (thin solid curve) for a 100-keV electron moving in front of 8 fcc(111) planes of air spherical voids of radius $r=418 \mathrm{~nm}$ in silicon $(\epsilon=11.9)$, where the lattice constant $a$ is $1222 \mathrm{~nm}$ (the filling fraction is $67 \%$ and the separation between sphere centers is $d=873 \mathrm{~nm}$ ). The electron is moving inside Si at a distance of $876 \mathrm{~nm}$ from the void spheres. (b) Transmittance for light inciding normally (thick curve) and with the Cherenkov angle $\theta_{c}=68.1^{\circ}$ (thin curve) for the same system. (c) Bulk band structure projected on the (111) plane for the same system, where the shaded zones indicate regions where no propagating electromagnetic waves are allowed. A full gap is observed in the range $\omega d / c \approx 3.23-3.35$.

FIG. 2: Frequency variation of the energy loss probability (thick solid curve), the reflected plus direct light emission probability (dashed curve) and the transmitted light emission probability (thin solid curve) for a 100-keV electron moving in front of 8 fcc(111) planes of air spherical voids of radius $r=418 \mathrm{~nm}$ in silicon $(\epsilon=11.9)$, where the lattice constant $a$ is $1222 \mathrm{~nm}$ (the filling fraction is $67 \%$ and the separation between sphere centers is $d=873$ $\mathrm{nm})$. The surface of the crystal is $131 \mathrm{~nm}$ from the last sphere. The electron is moving in air at a distance of $800 \mathrm{~nm}$ from this surface.

FIG. 3: Variation of the reflected plus direct emission probability (dashed curve) and the transmitted emission probability (solid curve) with the distance between a 100-keV electron and the first of the $8 \mathrm{fcc}(111)$ planes of air spherical voids of radius $r=418 \mathrm{~nm}$ in silicon, where the lattice constant $a$ is $1222 \mathrm{~nm}$. The emitted light wavelength is $\lambda=1.68 \mu \mathrm{m}$ (i.e, $\omega d / c=3.25)$. The asterisk $(*)$ corresponds to the electron-spheres distance of Fig. 1 (a). 


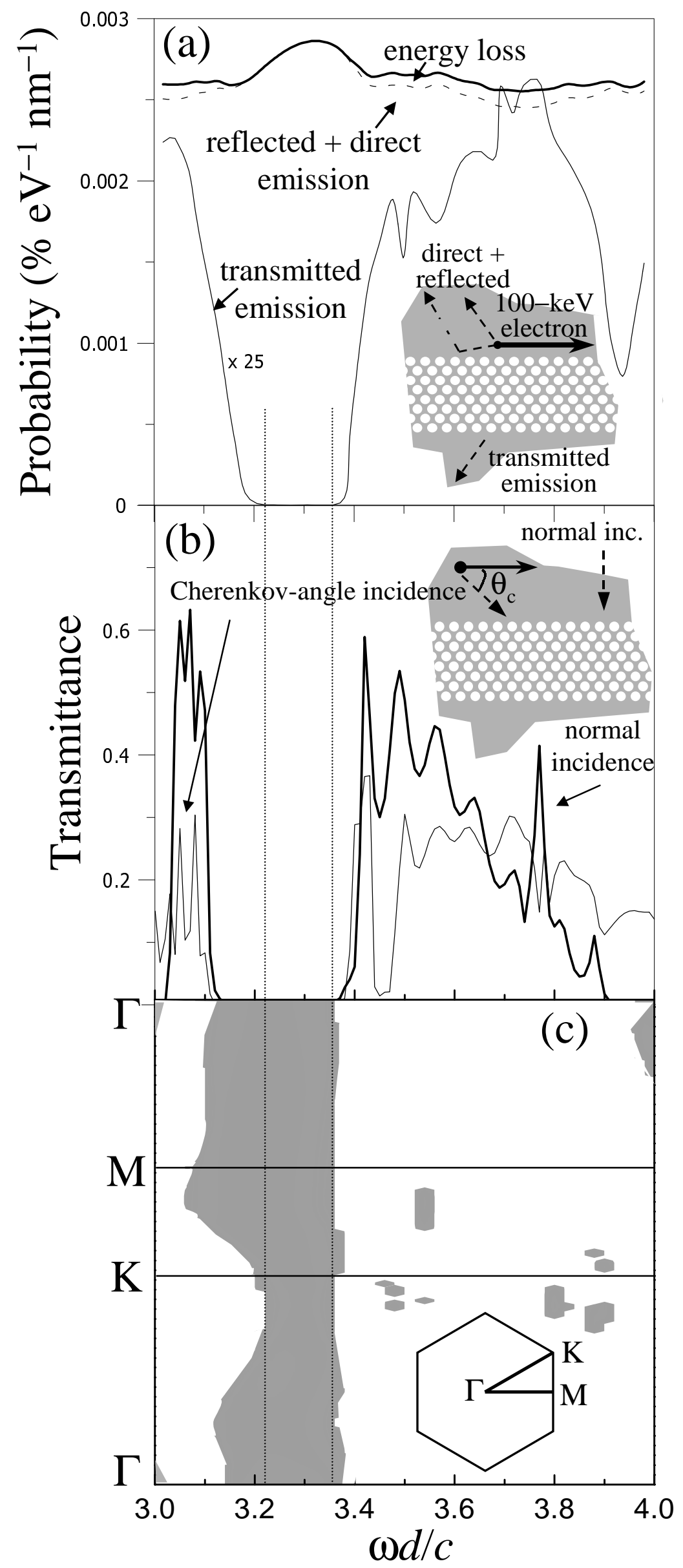

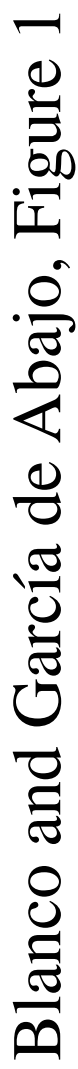




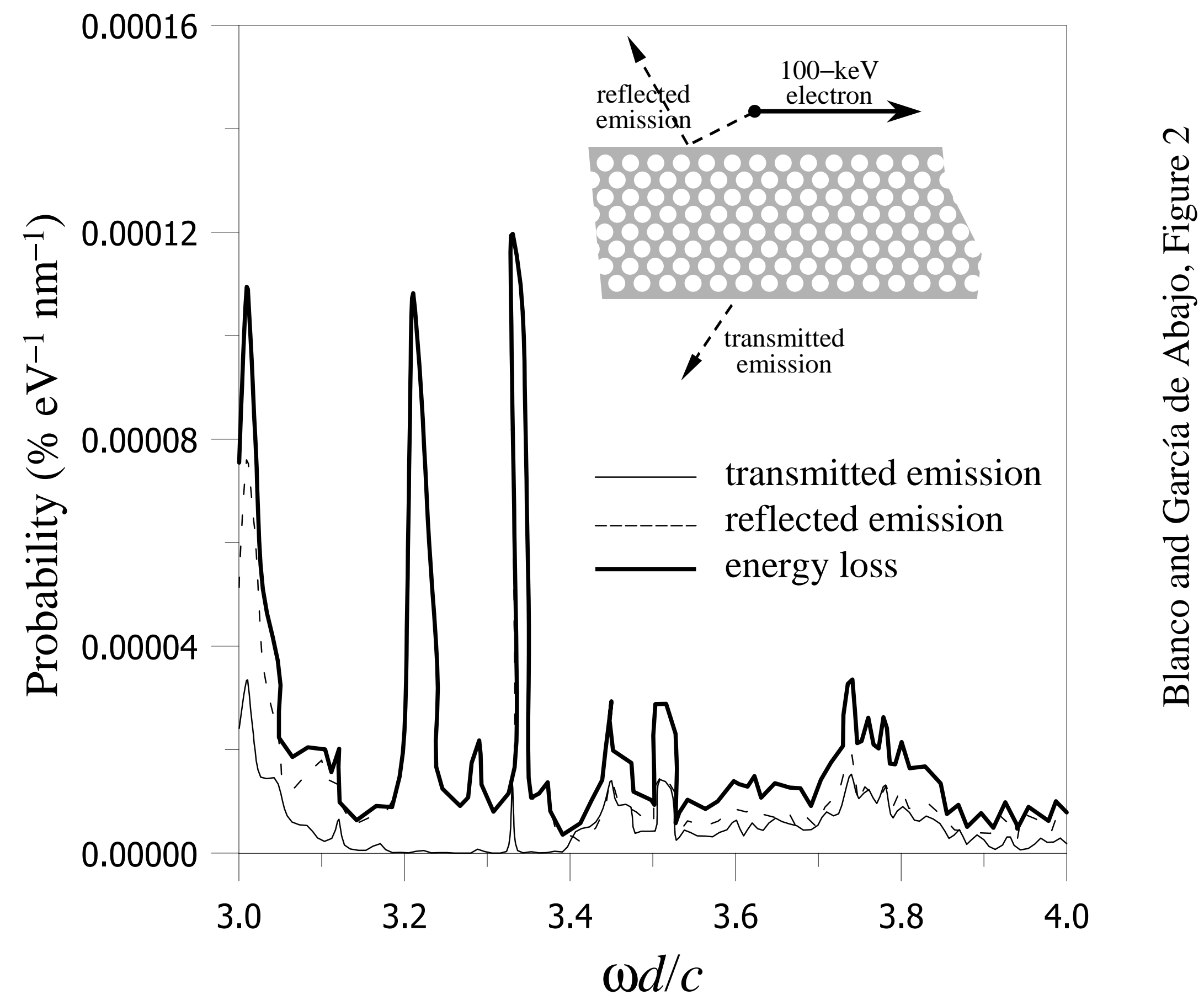




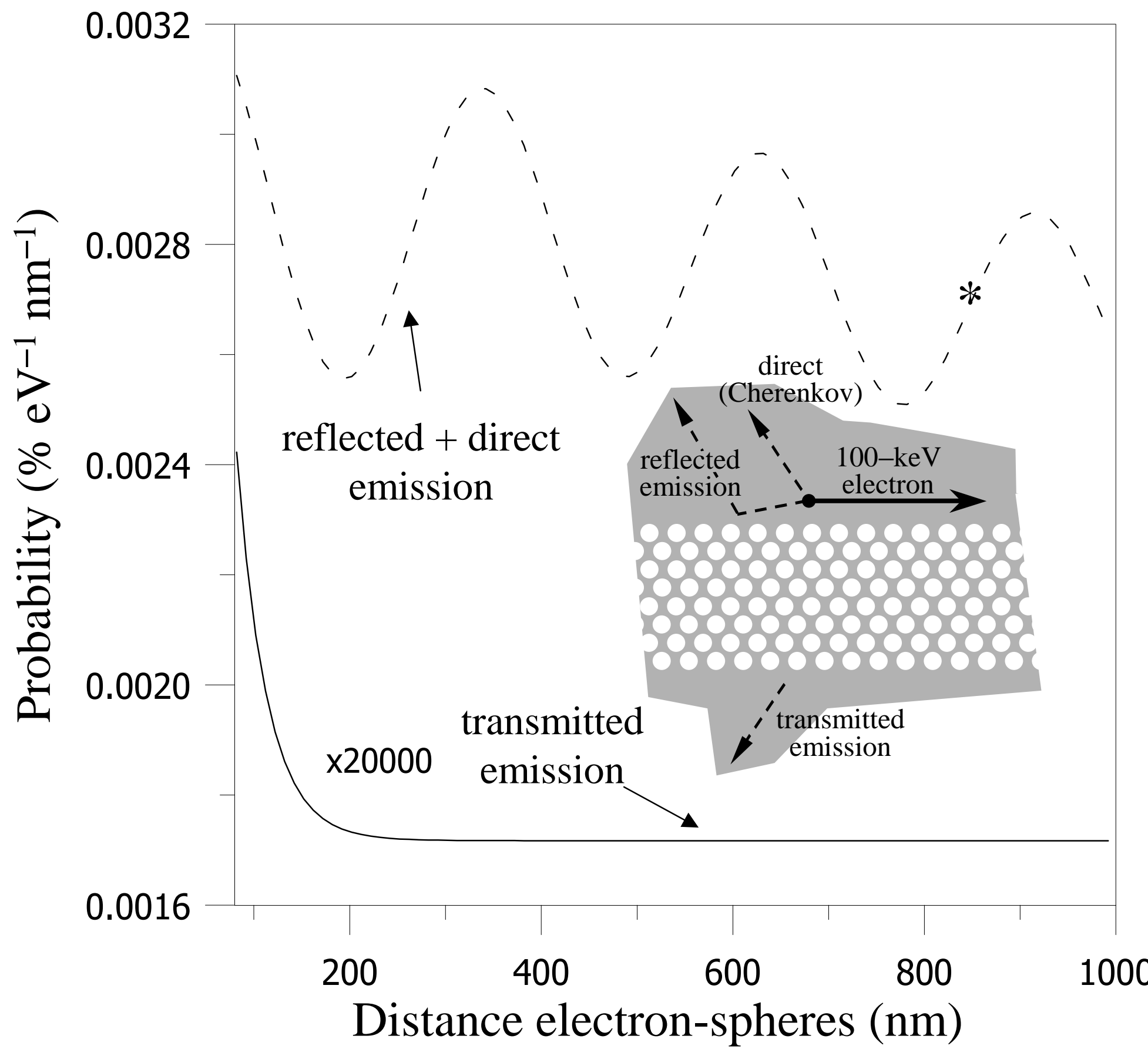

0
0
0
0
01
0
0
0
0
0
0
0
0
0
0
0
0
0
0
0
0
0
0 